\title{
Peripheral blood correlates of virologic relapse after Sofosbuvir and Ribavirin treatment of Genotype-1 HCV infection
}

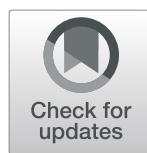

Cody Orr', Wenjie $\mathrm{Xu}^{2}$, Henry Masur ${ }^{3}$, Shyam Kottilil ${ }^{4}$ and Eric G. Meissner ${ }^{1,5^{*}}$ (i)

\begin{abstract}
Background: Treatment of chronic hepatitis $C$ virus infection with direct acting antiviral therapy results in viral elimination in over $90 \%$ of cases. The duration of treatment required to achieve cure differs between individuals and relapse can occur. We asked whether cellular and transcriptional profiling of peripheral blood collected during treatment could identify biomarkers predictive of treatment outcome.

Methods: We analyzed peripheral blood collected during treatment of genotype $1 \mathrm{HCV}$ with 24 weeks of sofosbuvir and weight-based or low dose ribavirin in a trial in which $29 \%$ of patients relapsed. Changes in host immunity during treatment were assessed by flow cytometry and whole blood gene expression profiling. Differences in expression of immune-relevant transcripts based on treatment outcome were analyzed using the Nanostring Human Immunology V2 panel.

Results: Multiple cellular populations changed during treatment, but pre-treatment neutrophil counts were lower and natural post-treatment killer cell counts were higher in patients who relapsed. Pre-treatment expression of genes associated with interferon-signaling, T-cell dysfunction, and T-cell co-stimulation differed by treatment outcome. We identified a pre- and post-treatment gene expression signature with high predictive capacity for distinguishing treatment outcome, but neither signature was sufficiently robust to suggest viability for clinical use.

Conclusions: Patients who relapse after hepatitis C virus therapy differ immunologically from non-relapsers based on expression of transcripts related to interferon signaling and T-cell dysfunction, as well as by peripheral neutrophil and NK-cell concentrations. These data provide insight into the host immunologic basis of relapse after DAA therapy for HCV and suggests mechanisms which may be relevant for understanding outcomes with currently approved regimens.
\end{abstract}

Keywords: Hepatitis C virus, Direct acting antiviral, Gene expression analysis, Sustained virologic response, Relapse

\footnotetext{
* Correspondence: meissner@musc.edu

${ }^{1}$ Division of Infectious Diseases, Medical University of South Carolina, 135 Rutledge Ave, MSC752, Charleston, SC 29425, USA

${ }^{5}$ Department of Microbiology and Immunology, Medical University of South Carolina, 135 Rutledge Ave, MSC752, Charleston, SC 29425, USA

Full list of author information is available at the end of the article
}

(c) The Author(s). 2020 Open Access This article is licensed under a Creative Commons Attribution 4.0 International License, which permits use, sharing, adaptation, distribution and reproduction in any medium or format, as long as you give appropriate credit to the original author(s) and the source, provide a link to the Creative Commons licence, and indicate if changes were made. The images or other third party material in this article are included in the article's Creative Commons licence, unless indicated otherwise in a credit line to the material. If material is not included in the article's Creative Commons licence and your intended use is not permitted by statutory regulation or exceeds the permitted use, you will need to obtain permission directly from the copyright holder. To view a copy of this licence, visit http://creativecommons.org/licenses/by/4.0/ The Creative Commons Public Domain Dedication waiver (http://creativecommons.org/publicdomain/zero/1.0/) applies to the data made available in this article, unless otherwise stated in a credit line to the data. 


\section{Background}

Over $90 \%$ of patients with chronic hepatitis $\mathrm{C}$ virus (HCV) infection achieve a sustained virologic response (SVR) after 8-12 weeks of treatment with currently approved direct acting antivirals (DAAs). When 4-6 week courses of treatment are given, treatment failures are unacceptably high. Such failures could relate to drug potency, drug susceptibility of the virus, virus replication levels, and patient adherence, but host factors may also play a role [1-6].

Although race, gender, liver disease stage, baseline viral load, viral genotype, and concomitant medications have correlated with population odds of achieving SVR in some studies, there are currently no clinically reliable predictors of treatment response [6]. Pre-treatment $\mathrm{HCV}$ resistance-associated variants (RAVs) associate with relapse with some regimens $[7,8]$, but existence of RAVs alone cannot predict outcomes in individuals and other factors must be involved $[4,9]$. We and others observed higher expression of functional markers of innate, interferon-related, and adaptive immune function in patients who achieved SVR with DAA therapy relative to patients who relapsed [10-16]. We thus hypothesize there is a role for host immunity in modulating, or at least reflecting, the odds of achieving SVR.

Specifically, our analysis of liver biopsies from HCV-infected patients treated with sofosbuvir and ribavirin identified higher post-treatment expression of interferon-related genes in patients achieving SVR, suggesting induction of endogenous type-I interferon activity during therapy may facilitate SVR [14]. In a separate study of PBMCs from two sofosbuvir-based DAA trials, we observed dynamic changes in the composition of peripheral blood during treatment [16], suggesting altered migration of peripheral immune cells during treatment may have relevance for understanding the role of intrahepatic and systemic immunity in viral clearance.

In this study, we combine these observations and ask whether immune markers in peripheral blood before and after treatment with sofosbuvir and ribavirin differed between patients achieving SVR vs. those who relapsed. Patient adherence to antiviral treatment in this trial did not differ based on treatment outcome, but relapse was common, occurring in $29 \%$ of subjects [17]. The regimen of sofosbuvir and ribavirin for the treatment of genotype 1 $\mathrm{HCV}$ infection is no longer in clinical use due to unacceptably high rates of relapse relative to currently approved therapies. Because relapse was common in this trial, close analysis of longitudinal samples collected during the trial presented an opportunity to understand the mechanistic underpinnings of virologic relapse, which are likely relevant for understanding why relapse can occur with currently approved regimens, albeit less frequently.

\section{Methods}

\section{Clinical cohort}

Clinical samples collected as part of the SPARE clinical trial (NCT01441180) were used in this study. SPARE was a trial conducted at the National Institute of Allergy and Infectious Diseases (NIAID) in which patients infected with HCV genotype-1 were treated with sofosbuvir and weight-based or low dose ribavirin for 24 weeks, as previously described $[14,17]$. This study received approval from the Institutional Review Boards at NIAID and the Medical University of South Carolina and was conducted in concordance with the 1975 Declaration of Helsinki. All patients provided written informed consent, as previously reported with initial publication of the clinical trial result [17], which included permissions to use samples and data collected in the trial for future studies.

\section{Flow cytometry}

Immuno-phenotyping of peripheral blood drawn into EDTA-containing blood collection tubes was performed using a modification of the Centers for Disease Control and Prevention guidelines in a Clinical Laboratory Improvement Act-certified laboratory, as previously described [16]. Cells were stained with combinations of monoclonal antibodies and then lysed with Optilyse-C (Beckman Coulter, Hialeah, FL), washed twice, and resuspended in $500 \mu \mathrm{l}$ of phosphate-buffered saline (Cambrex, Walkersville, MD). Samples were analyzed immediately on a Becton Dickinson FacsCanto flow cytometer (BDBiosciences, San Jose, CA). 4-color antibody panels used for cellular identification and enumeration have been previously described [16].

Lymphocytes were identified by side scatter and CD45 staining, with confirmation that less than $5 \%$ of cells within the lymphocyte gate expressed CD14 [18]. Absolute concentrations of specific lymphocyte populations (CD3+, CD4+, CD8+, CD4+/8+, CD19, and CD16+/56+ lymphocytes) were calculated using the measured percentage of each cell type within the lymphocyte gate and the absolute lymphocyte concentration [16].

\section{Gene expression analysis}

Whole blood was collected into PaxGene RNA tubes at the time of venipuncture either pre-treatment or at the end of treatment, prior to virologic relapse, and was stored at $-80^{\circ} \mathrm{C}$ until processing. Paired blood samples were available from 40 subjects enrolled in the trial, including 24 who achieved SVR and 16 who relapsed. RNA was extracted using the PAXgene Blood miRNA Kit (Qiagen) according to the manufacturer's instructions. RNA quality was assessed using the Agilent Bioanalyzer. RNA samples had a median RNA integrity number (RIN) of 7.2 and most (91\%) had an RIN greater than 6.5. 
A Nanostring nCounter was used to directly quantitate expression of 579 immune transcripts (Human Immunology v2 Panel). The lower limit of detection is 20 counts, and as such, counts below 25 were considered to be background signal and counts below 100 were considered to be semi-quantitative. Genes in which $30 \%$ or more of samples had a count under 100 were excluded from subsequent analysis. Normalization of gene expression was performed relative to housekeeping genes using the GeNorm algorithm. Of 15 housekeeping genes included on the panel, 11 with the lowest standard deviation after normalization were used (all with $\mathrm{SD}<0.30$ ). Data were Z-score transformed and grouped by unsupervised clustering. Mean Square Error analysis identified 4 samples with low data quality, and as such these and their paired samples were excluded from further data analysis, yielding a final dataset of 36 paired samples ( $n=23$ SVR, $n=13$ relapse). Demographic characteristics of these 36 patients are shown in Table 1. More subjects had advanced liver fibrosis (HAI fibrosis $3-4$ ) in the relapse group $(n=8)$ than the SVR group $(n=5)$. Validation of expression for select genes was performed using quantitative reverse transcriptase-polymerase chain reaction (qRT-PCR), as previously described [14].

\section{Immune cell profiling}

Advanced Analysis Immune Cell Type Profiling was performed with nSolver software to predict changes in cell type frequencies based on measured expression of gene sets validated to be cell-type specific (Supplemental Table 1) and was compared to data independently derived by flow cytometry [19].

\section{Statistical analysis}

All statistical analyses on NanoString data were performed on $\log 2$ transformed normalized counts. Differential expression analyses were carried out using nSolver4.0 and Advanced Analysis package 2.0 (NanoString ${ }^{\mathrm{tm}}$ ), following the default analysis pipeline. A machine learning algorithm based on Elastic Net, a regression method that applies both the lasso and ridge penalties, was used to discover gene expression signatures that differentiate relapse and SVR patients both pre- and post-treatment. Curated, $\log 2$ transformed expression data was used to generate a linear predictor score $=\Sigma X i \beta i(X=$ gene $X$ expression level; $\beta=$ coefficient). Cross-validation runs were performed to select the strength of each penalty and for estimation of AUC based on the training data set. Demographic and flow cytometry data were analyzed by Chi-square or t-test methods as indicated.

\section{Results}

To test the hypothesis that immune differences during DAA treatment associate with treatment outcome, we analyzed peripheral blood from the SPARE trial in which a significant number of patients experienced treatment relapse (29\%) [17]. We first examined changes in the cellular composition of peripheral blood over the course of treatment by flow cytometry. We observed a significant decrease in the concentration of total lymphocytes, NKcells, B-cells, CD4+ T-cells, and CD8+ T-cell during treatment when considering the entire patient cohort (Fig. 1a). We had not observed these declines in data from previously published ribavirin-free DAA cohorts [16], and suspect they may reflect an impact of ribavirin on overall leukocyte counts, as previously reported [20]. We also observed a significant relative increase in neutrophil and CD14+ monocytes cell concentrations over the course of treatment (Fig. 1a).

We next analyzed predicted changes in cellular concentrations calculated by transcriptional analysis of whole blood using the Nanostring Immune Cell Type Profiling platform. Nearly identical results were obtained, as B-cells, NK CD56-dim cells, total T-cells, Th1 cells, cytotoxic CD8+ T-cells, and exhausted CD8+ cells were all predicted to decrease over the course of treatment, while CD45+ cells (a leukocyte marker) and neutrophils were predicted to increase (Fig. 1b). The similarity of findings derived by different techniques suggests whole blood transcriptional profiling was a valid approach to provide meaningful and biologically relevant insight.

We next examined whether cellular populations differed pre- or post-treatment based on outcome. Pretreatment, only neutrophil concentration differed by

Table 1 Demographic Profile of Patients Included in Whole Blood Transcriptional Analysis

\begin{tabular}{llll}
\hline & SVR $(\boldsymbol{n}=\mathbf{2 3})$ & Relapse $(\boldsymbol{n}=\mathbf{1 3})$ & \\
\hline Ribavirin Dose (Low/High) & $9 / 14$ & $9 / 4$ & $p=0.04^{\mathrm{a}}$ \\
Gender (M/F) & $13 / 10$ & $11 / 2$ & $p=0.08^{\mathrm{a}}$ \\
BMI mean (range) & $31(23-46)$ & $29(19-43)$ & $p=0.39^{\mathrm{b}}$ \\
HAl Fibrosis mean (range) & $1.17(0-3)$ & $2.38(0-4)$ & $p=0.004^{\mathrm{b}}$ \\
Pre-treatment ALT mean (range) & $51(28-141)$ & $58(31-155)$ & $p=0.88^{\mathrm{b}}$ \\
\hline
\end{tabular}

$B M I$ body mass index, HAI Histologic Activity Index, ALT Alanine aminotransferase

${ }^{a}$ statistical analysis by Chi-square test

${ }^{\mathrm{b}}$ statistical analysis by unpaired t-test 


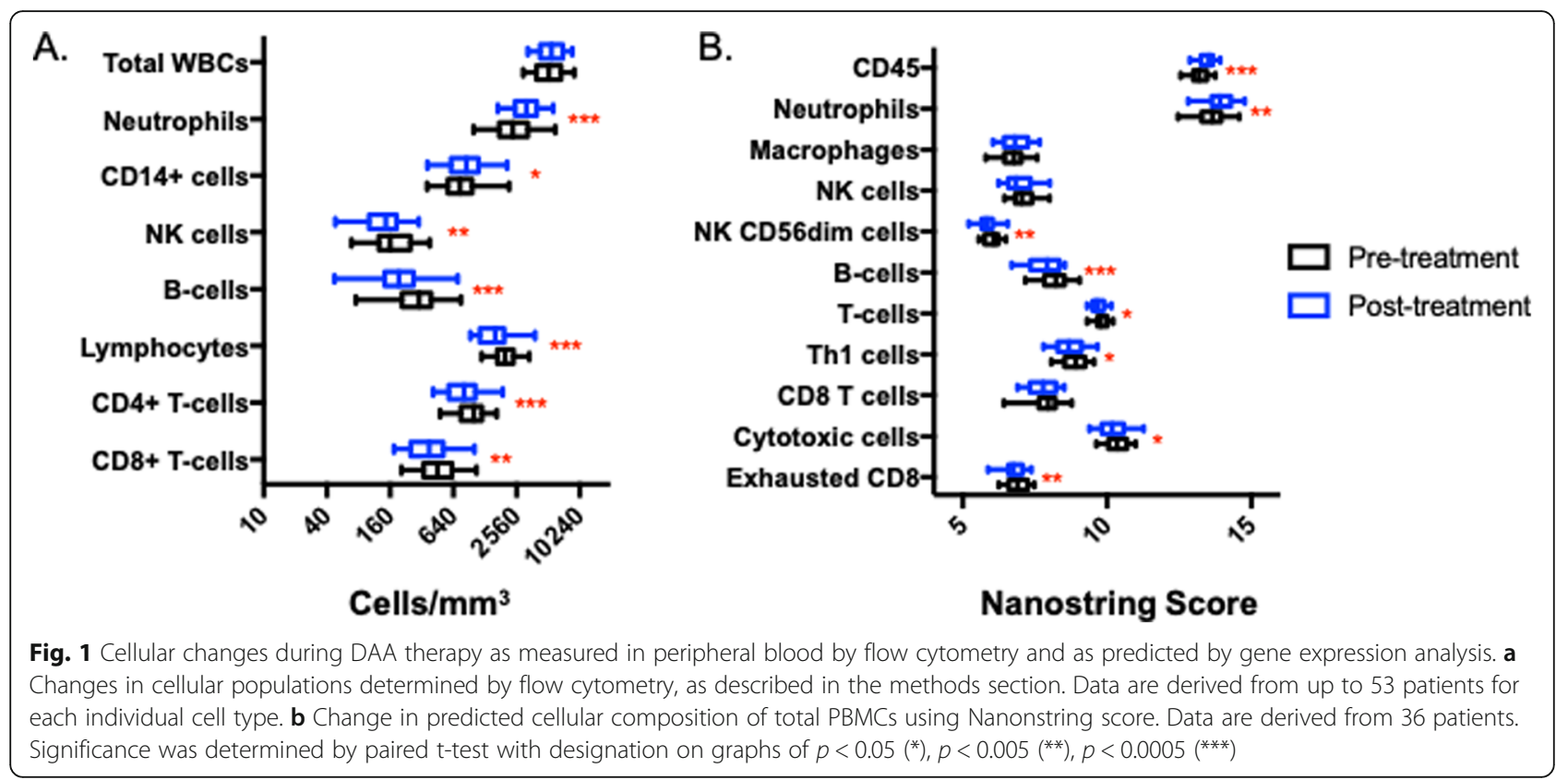

outcome, with relapsers having lower neutrophil concentration as assessed by both flow cytometry and gene expression profiling (Fig. 2a). Post-treatment, relapsers had higher concentrations of NK-cells by flow cytometry (Fig. 2b), but no other significant differences (data not shown). By gene expression profiling, relapsers had higher predicted concentrations of NK cells and cytotoxic cells, and a trend towards higher concentrations of total CD8+ Tcells and exhausted CD8+ T-cells (Fig. 2b).

We next analyzed expression of individual transcripts and examined changes with treatment and differences by outcome. In the entire patient cohort, 208 genes changed significantly during treatment (Fig. 3a, Supplemental Excel File Tab 1), a substantial percentage of the total genes tested. Multiple ISGs decreased with treatment (e.g. MX1, IFIT2, CXCL10, RARRES3), consistent with prior results from other DAA trials [14, 21, 22]. IFNAR1 and IFNAR2, which comprise the type-I interferon receptor, both had increased expression posttreatment. Genes associated with T-cell activation (e.g. CD80, CD160, LAG3, BTLA, ICOS) decreased with treatment. An analysis of the data by hierarchical clustering to examine any impact of gender, IFNL4 genotype, fibrosis stage, ribavirin dose, and pre-treatment ALT levels,

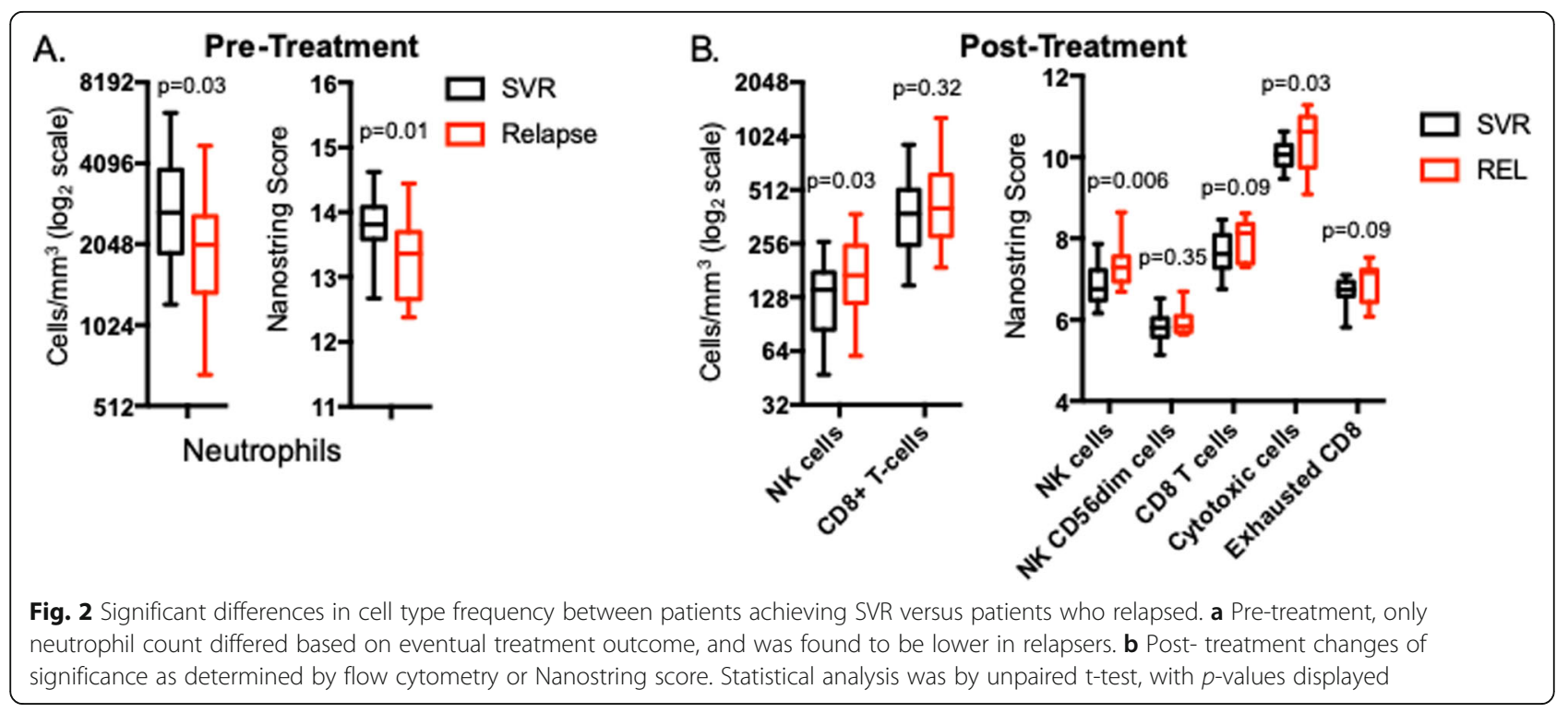




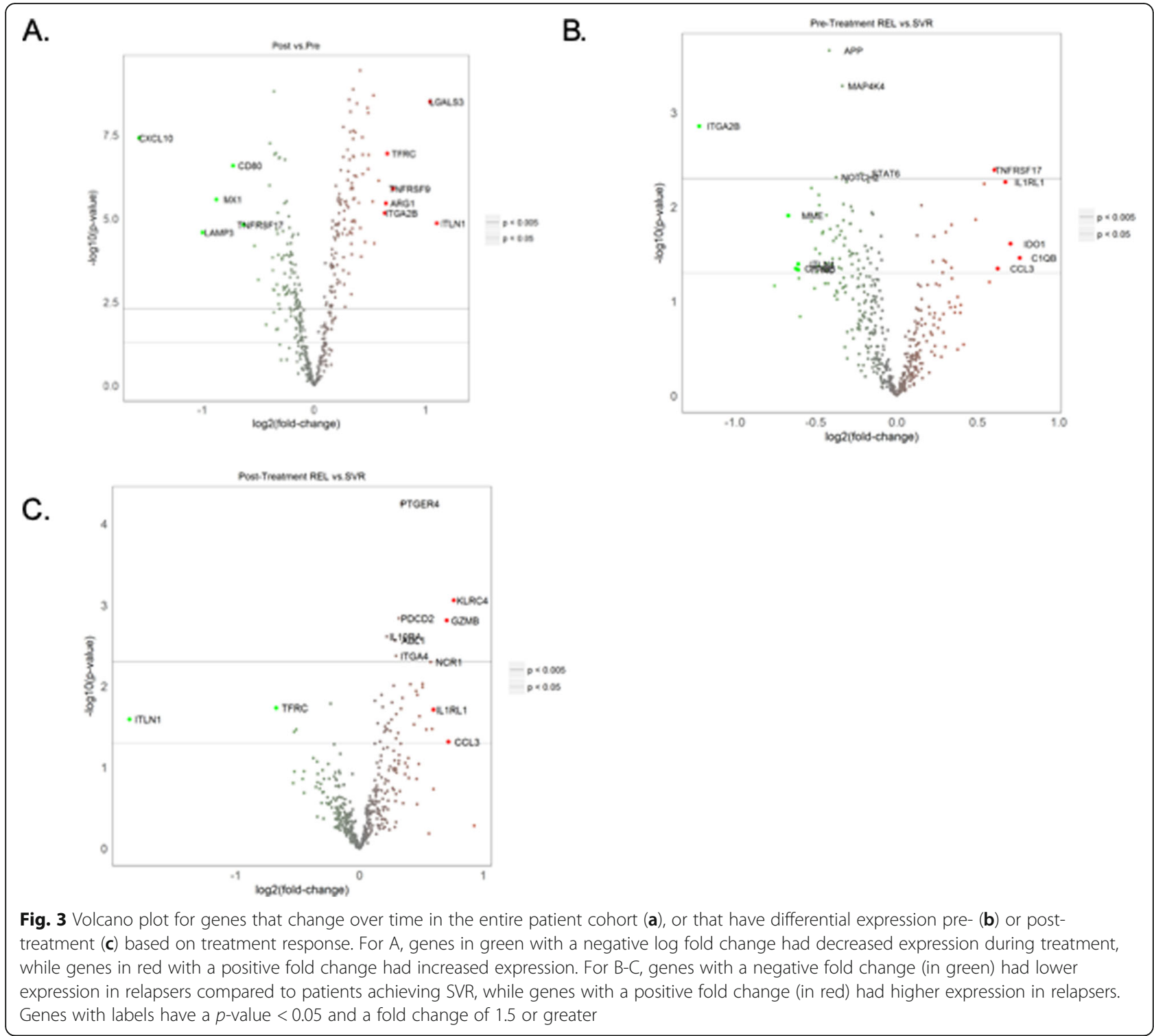

several of which differed based on outcome in this patient cohort (Table 1), did not identify clear clustering based on these factors (Supplemental Fig. 1).

We next analyzed differences by treatment outcome and identified 84 genes pre-treatment and 43 genes post-treatment with differential expression (Fig. 3b-c, Supplemental Excel File Tabs 2-3). Significant genes with the highest fold differential expression between SVR and relapse patients pre- and post-treatment are displayed as heatmaps (Fig. 4).

Pre-treatment, prior to DAA exposure, a number of genes related to TLR-mediated pathogen recognition (TLR8, TLR2, TLR5, IRAK4, TOLLIP), IL1 signaling, complement activation, and TNF signaling had lower expression in patients who later experienced treatment relapse. Multiple genes related to neutrophils (FCGR3A/B,
FCGR2A, FCGR2A/C) were lower in relapsers, consistent with observations derived by flow cytometry. Interestingly, pre-treatment expression of IFNAR1, IFNAR2, and SOCS3 was lower in relapsers, while pre-treatment expression of SOCS1 was higher (Fig. 4a and Supplemental Excel File Tab. 2). SOCS3 and SOCS1 are known negative regulators of interferon signaling. Several genes associated with T-cell dysfunction/tolerance (CTLA4$T M, C D 244, I D O 1)$ had higher pre-treatment in patients who subsequently relapsed.

Post-treatment, several consistent changes distinguished the groups by outcome. First, patients who relapsed had higher expression of genes related to NK-cell frequency and function (KLRK1, KLRD1, KLRG1, NCR1, KLRC3, KLRC4, PRF1, GZMB), which includes both activating and inhibitory receptors, consistent with data 


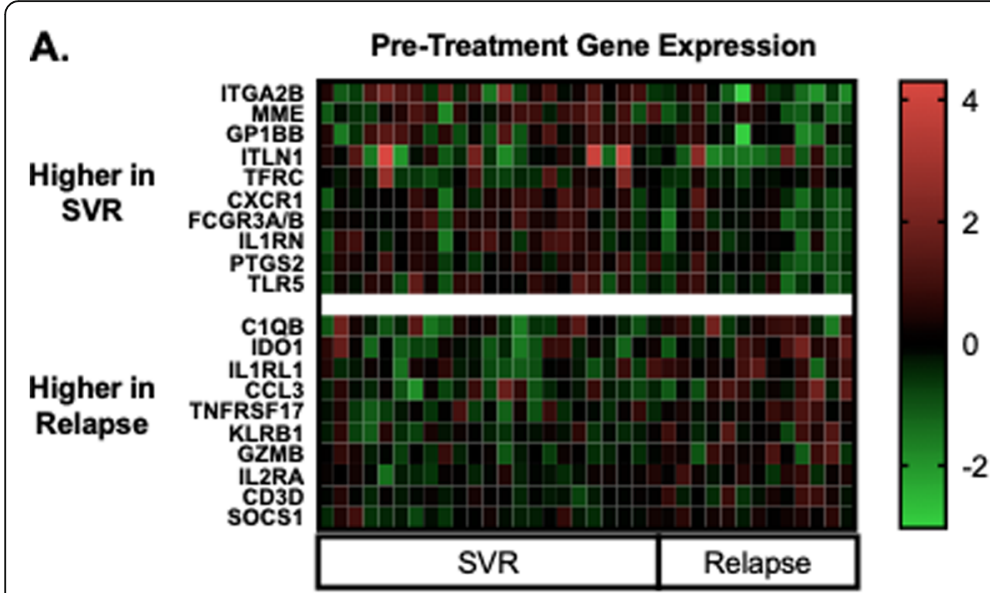

B.

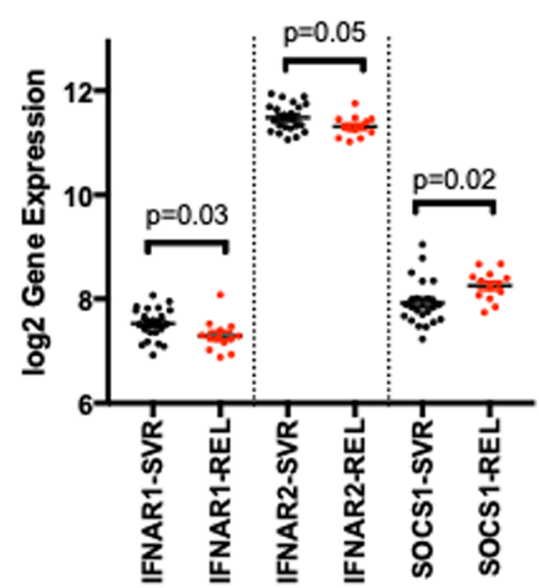

C.

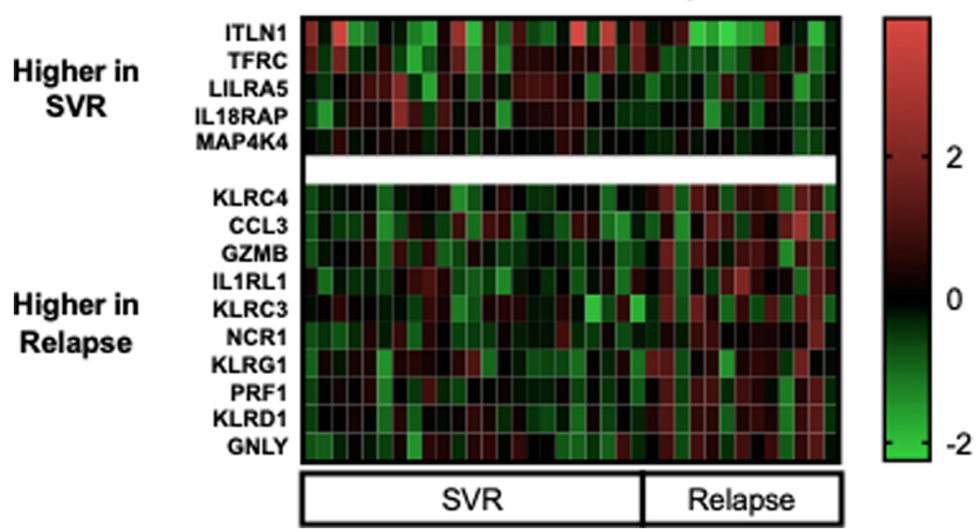

Fig. 4 Genes with the highest differential expression between SVR and relapse in whole blood either pre- (a-b) or post- treatment (c). Delta log2 gene expression was determined for each patient in relation the mean expression for the cohort and all displayed genes achieved significance. $P$ values for (b) were determined by unpaired t-test

derived by flow cytometry. In addition, patients who relapsed had higher expression of genes associated with Tcell dysfunction (HAVCR2, KLRG1, CD244).

Finally, we asked whether expression of any gene or combination of genes pre-or post-treatment could have sufficient capacity to robustly predict clinical outcome. Using a machine learning algorithm, we identified a 12-gene pre-treatment and an 5-gene post-treatment set with high predictive capacity in training sets with AUCs of 0.95 and 0.86, respectively (Supplemental Figs. 2-3). As we lacked data from an independent cohort to validate these gene signatures, we performed an internal validation analysis and found neither signature had a consistently high AUC on cross-validation runs, both with mean AUCs of 0.66 (Supplemental Figs. 2-3).

\section{Discussion}

This study asked whether analysis of routinely available clinical samples could distinguish HCV patients who relapse or achieve SVR after treatment with sofosbuvir and ribavirin and provide insight into mechanisms of relapse. While the antiviral regimen of sofosbuvir and ribavirin is no longer in routine clinical use, the high rates of relapse that occurred in the study offered an opportunity to identify biologic correlates of relapse that likely remain relevant when trying to understand mechanisms of relapse that occur with currently approved DAA regimens. We identified differences in neutrophil count pretreatment and NK cell count post-treatment that distinguish patients by outcome. In addition, whole blood expression of transcripts related to interferon signaling, $\mathrm{T}$ cell dysfunction, and NK-cells distinguished patients by treatment outcome. These data provide evidence that heightened IFN signaling and dysregulated adaptive 
immunity pre-treatment associate with higher odds of relapse.

Pre-treatment, our primary observation was that neutrophil count was lower in eventual relapsers and that expression of genes related to pathogen detection, neutrophil function, and interferon signaling differed by outcome. While multiple canonical ISGs decreased during treatment and did not differ by treatment outcome (e.g. CXCL10, RARRES3, MX1), we were intrigued to find that expression of both IFNAR1 and IFNAR2 increased with treatment, suggesting an inverse correlation between type-I receptor expression level and interferon signaling. These data are consistent with previous reports that IFNAR1 gene expression is reduced in PBMC of chronic HCV patients [23]. Our observation that patients who relapsed had lower baseline expression of both IFNAR1 and IFNAR2 thus suggests a heightened state of interferon signaling pre-treatment. This observation suggests that, as was trued for interferon-based therapy [24], heightened interferon signaling pretreatment may reflect a poor antiviral immune state less amenable to $\mathrm{HCV}$ clearance, and thus more prone to relapse.

Although type-I and type-III IFN signaling induce a similar ISG transcriptional profile in cells expressing both receptors, the pattern of genes is not identical and the kinetics of induction can differ [25]. As noted above, the expression of SOCS1, a negative regulator of IFN signaling, decreased during treatment, consistent with the pattern observed for other ISGs. Expression was higher pre-treatment in relapsers, which was the inverse pattern of that observed with IFNAR1 and IFNAR2. This data is intriguing as type-III IFN signaling has previously been shown to selectively induce SOCS1 relative to type-I IFN signaling in human and chimpanzee liver [26, 27], suggesting the chronic IFN signature in HCV patients could relate to type-III IFN signaling. Whether the lower neutrophil count we observed pre-treatment in relapsers reflects higher levels of endogenous interferon signaling, as interferon treatment causes a relative neutropenia [28, 29], requires further study. This observation regarding neutrophils, which contribute significantly to the cellular composition of whole blood analyzed by gene expression profiling, is of particular interest as neutrophils are excluded from most analyses examining PBMCs. Taken together, these data suggest higher baseline IFN activation in patients who subsequently relapsed.

Post-treatment, relapsers had higher levels of NK cells and NK-cell associated transcripts than patients achieving SVR. This is intriguing as changes in NK cell function and frequency during DAA therapy have been previously reported, with most observing decreases during $\mathrm{HCV}$ treatment, including reduced surface expression of NKp30 (NCR3), NKp46 (NCR1), TRAIL, and
NKG2A [10, 30]. Our data suggests NK-cell frequency and relative activation state post-treatment could reflect presence of residual intrahepatic $\mathrm{HCV}$. While NKp30 and NKG2A were not analyzed in this study, we found no change in TRAIL or NKp46 expression during treatment, but did find higher expression of the activating receptor NKp46 post-treatment in relapsers. Our data are also intriguing in the light that IFNL genotype and IFN activity have been linked to differential NK cell activation [31-34]. As understanding NK-cell functional status from whole blood gene expression profiling is challenging, our data support future experiments focused on analysis of NK-cell phenotype at the end of treatment.

Another major finding of the study relates to genes associated with T-cell dysfunction. We observed significantly higher post-treatment expression of markers of T-cell exhaustion in relapsers including HAVCR2 (TIM3) and KLRG1 (Fig. 4) [35] .While CTLA-4-TM decreased over the course of treatment in the entire cohort, relapsers had higher pre-treatment expression. In addition, while 2B4 (CD244) did not change during treatment, patients who relapsed had higher expression both pre- and post-treatment. Taken together, these data associate relapse with $\mathrm{T}$-cell dysfunction, consistent with prior findings derived by flow cytometry [10-15]. Prior work identified a heterogenous response to in vitro blockade of inhibitory co-stimulatory receptors (PD1, CTLA4, TIM3, 2B4) for the CD8 response in patients with $\mathrm{HCV}$ infection [36], and may reflect distinct ways in which individual hosts respond to the same pathogen, which could have relevance for treatment relapse. In our study we observed no change in PDL1 (CD274) during treatment and no difference pre- or post-treatment based on treatment outcome, while expression of PD1 (PDCD1) was low and did not satisfy the stringency criteria of this analysis. As IFN-driven signaling can cause T-cell exhaustion, which can be reversed with IFN blockade [37], our data suggesting heightened interferon signaling pre-treatment in relapsers may thus relate mechanistically to parallel observations of higher T-cell dysfunction in relapsers.

Together, these data suggest relapsers might be more likely to respond if they could be identified prospectively, and if the rate of their immune restoration on DAA therapy could be identified and considered when considering treatment duration. Given the high efficacy of currently approved DAA regimens, it is intriguing to consider that subjects experiencing more rapid immune "rejuvenation" could achieve cure with shortened treatment durations, while those with a more sluggish immune response on therapy may benefit from a longer course of treatment to minimize the risk of relapse. When considering other regulators of $\mathrm{T}$-cell activation [38], we did observe down-regulation of CD80, CD160, 
LAG3, BTLA, and ICOS with treatment (Fig. 4, Supplemental Excel File Tab 1), but saw no difference at any time point based on treatment outcome.

A limitation of our study is that most transcripts are not cell-type specific. As such our suggestions regarding mechanism and causation require prospective validation. Other limitations include the low number of patients with IFNL4 genotype predicting inability to produce IFNL4 protein, limiting our capacity to analyze difference in gene expression patterns attributable to this. Although more patients with advanced fibrosis were present in the group of patients who relapsed (Table 1), which could have confounded our analysis, we did not identify an association of fibrosis with the gene expression patterns that correlated with relapse in this study. While we did not identify an impact of ribavirin dose on the reported results, because ribavirin can impact gene expression and impart sensitization to IFN signaling [39] an impact of ribavirin dose on these findings cannot be ruled out [17]. Finally, as discussed above, sofosbuvir and ribavirin is not currently in clinical use for treatment of genotype-1 HCV infection due to high rates of relapse. Nonetheless, the biologic correlates identified in this study likely have relevance for understanding why relapse still occurs in a subset of subjects receiving current regimens, and as such these findings will benefit from further validation using samples from cohorts receiving ribavirin-free regimens.

\section{Conclusions}

In conclusion, neutrophil count pre-treatment and NKcell count post-treatment distinguish patients achieving SVR from those who relapse after sofosbuvir and ribavirin treatment. In addition, expression of interferon related genes and genes related to T-cell dysfunction differed by outcome, supporting a role for host immunity in modulating $\mathrm{HCV}$ treatment outcome with DAA therapy. These findings suggest that favorable host immunity could enable higher response rates and shorter durations of therapy, which could have significant economic and adherence implications.

\section{Supplementary Information}

The online version contains supplementary material available at https://doi. org/10.1186/s12879-020-05657-5.

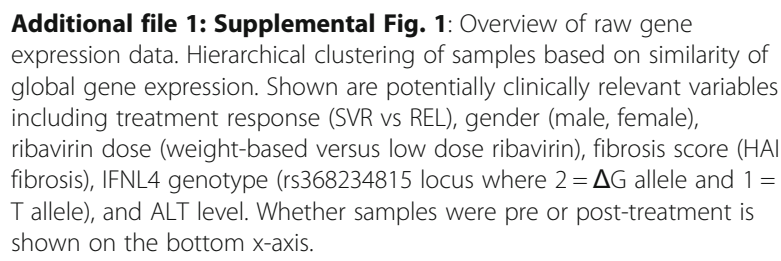

Additional file 2: Supplemental Fig. 2: Predictive capacity of genes expressed pre-treatment. Shown is a heatmap of genes identified by machine learning algorithm to best associate with outcome, performance of the training gene set by predictive score and AUC, and performance of the gene set when the data were re-analyzed with by cross-validation runs.

Additional file 3: Supplemental Fig. 3: Predictive capacity of genes expressed post-treatment. Shown is a heatmap of genes identified by machine learning algorithm to best associate with outcome, performance of the training gene set by predictive score and AUC, and performance of the gene set when the data were re-analyzed with by cross-validation runs.

Additional file 4: Supplemental Table 1: Genes on Nanostring Immunology panel used to assess cell-type frequency in whole blood.

Additional file $\mathbf{5}$.

\section{Abbreviations}

DAA: Direct acting antiviral; HCV: Hepatitis C virus; NIAID: National Institute of Allergy and Infectious Diseases; NK-cell: Natural Killer cell; PBMCs: Peripheral blood mononuclear cells; qRT-PCR: Quantitative reverse transcriptase-

polymerase chain reaction; RAV: Resistance associated variant; SVR: Sustained Virologic Response

\section{Acknowledgements}

Acknowledgments: The authors would like to thank the investigators and participants of the clinical trial from which the samples analyzed in this study were derived.

\section{Authors' contributions}

$\mathrm{CO}$ and EGM conducted the experiments. HM, SK, and EGM designed the study. WX performed the statistical analysis. All authors contributed to the drafting and editing of the manuscript.

\section{Author's information}

Not applicable

\section{Funding}

This study was funded by the National Institute of Allergy and Infectious Diseases grant K08Al121348 (EGM). This project was also funded in part with federal funds from the National Cancer Institute, National Institutes of Health, under Contract No. HHSN261200800001E, the National Institute of Allergy and Infectious Diseases, and the NIH Critical Care Medicine Department. The content of this publication does not necessarily reflect the views or policies of the Department of Health and Human Services, nor does mention of trade names, commercial products, or organizations imply endorsement by the U.S. Government.

\section{Availability of data and materials}

The datasets used and/or analyzed during the current study are available from the corresponding author on reasonable request.

\section{Ethics approval and consent to participate}

This study received approval from the Institutional Review Boards at NIAID and the Medical University of South Carolina and was conducted in concordance with the 1975 Declaration of Helsinki. All patients provided written informed consent, as previously reported with initial publication of the clinical trial result [17], which included permissions to use samples and data collected in the trial for future studies.

Consent for publication

Not applicable.

\section{Competing interests}

Wenjie Xu is an employee of Nanostring Technologies. Authors CO, SK, HM, and EGM declare they have no conflict of interest.

\section{Author details}

'Division of Infectious Diseases, Medical University of South Carolina, 135 Rutledge Ave, MSC752, Charleston, SC 29425, USA. ${ }^{2}$ Nanostring Technologies, Seattle, WA, USA. ${ }^{3}$ Critical Care Medicine Department, NIH Clinical Center, National Institutes of Health, Bethesda, MD, USA. ${ }^{4}$ Division of Clinical Care 
and Research, Institute of Human Virology, University of Maryland School of Medicine, Baltimore, MD, USA. ${ }^{5}$ Department of Microbiology and Immunology, Medical University of South Carolina, 135 Rutledge Ave, MSC752, Charleston, SC 29425, USA.

\section{Received: 26 November 2019 Accepted: 24 November 2020} Published online: 04 December 2020

\section{References}

1. Lawitz E, Poordad F, Gutierrez JA, Wells JT, Landaverde CE, Evans B, et al. Short-duration treatment with elbasvir/grazoprevir and sofosbuvir for hepatitis C: a randomized trial. Hepatology. 2017;65(2):439-

2. Lawitz E, Reau N, Hinestrosa F, Rabinovitz M, Schiff E, Sheikh A, et al. Efficacy of Sofosbuvir, Velpatasvir, and GS-9857 in patients with genotype 1 hepatitis $C$ virus infection in an open-label, phase 2 trial. Gastroenterology. 2016; 151(5):893-901 e1.

3. Kattakuzhy S, Wilson E, Sidharthan S, Sims Z, McLaughlin M, Price A, et al. Moderate sustained Virologic response rates with 6-week combination directly acting anti-hepatitis C virus therapy in patients with advanced liver disease. Clin Infect Dis. 2016:62(4):440-7.

4. Kohli A, Kattakuzhy S, Sidharthan S, Nelson A, McLaughlin M, Seamon C, et al. Four-week direct-acting antiviral regimens in noncirrhotic patients with hepatitis C virus genotype 1 infection: an open-label, Nonrandomized Trial. Ann Intern Med. 2015;163(12):899-907.

5. Lau G, Benhamou Y, Chen G, Li J, Shao Q, Ji D, et al. Efficacy and safety of 3-week response-guided triple direct-acting antiviral therapy for chronic hepatitis C infection: a phase 2, open-label, proof-of-concept study. Lancet Gastroenterol Hepatol. 2016:1(2):97-104.

6. Sarrazin C. The importance of resistance to direct antiviral drugs in HCV infection in clinical practice. J Hepatol. 2016:64(2):486-504.

7. Komatsu TE, Boyd S, Sherwat A, Tracy L, Naeger LK, O'Rear JJ, et al. Regulatory analysis of effects of hepatitis C virus NS5A polymorphisms on efficacy of Elbasvir and Grazoprevir. Gastroenterology. 2017;152(3):586-97.

8. Kish T, Aziz A, Sorio M. Hepatitis $C$ in a new era: a review of current therapies. P T. 2017;42(5):316-29.

9. Feld JJ, Jacobson IM, Hezode C, Asselah T, Ruane PJ, Gruener N, et al. Sofosbuvir and Velpatasvir for HCV genotype 1, 2, 4, 5, and 6 infection. N Engl J Med. 2015;373(27):2599-607.

10. Spaan M, van Oord G, Kreefft K, Hou J, Hansen BE, Janssen HL, et al. Immunological analysis during interferon-free therapy for chronic hepatitis C virus infection reveals modulation of the natural killer cell compartment. J Infect Dis. 2016;213(2):216-23.

11. Shrivastava S, Wilson E, Poonia B, Tang L, Osinusi A, Kohli A, et al. Augmentation of HCV specific immunity and sustained virological response (SVR). J Viral Hepat. 2017:24(9):742-9.

12. Ramamurthy N, Marchi E, Ansari MA, Pedergnana V, McLean A, Hudson E, et al. Impact of IFNL4 genotype on interferon-stimulated gene expression during DAA therapy for hepatitis C. Hepatology. 2018;68(3):859-71.

13. Carlton-Smith C, Holmes JA, Naggie S, Lidofsky A, Lauer GM, Kim AY, et al. IFN-free therapy is associated with restoration of type I IFN response in HIV1 patients with acute HCV infection who achieve SVR. J Viral Hepat. 2018; 25(5):465-72.

14. Meissner EG, Wu D, Osinusi A, Bon D, Virtaneva $K$, Sturdevant $D$, et al. Endogenous intrahepatic IFNs and association with IFN-free HCV treatment outcome. J Clin Invest. 2014;124(8):3352-63.

15. Meissner EG, Kohli A, Virtaneva K, Sturdevant D, Martens C, Porcella SF, et al. Achieving sustained virologic response after interferon-free hepatitis $C$ virus treatment correlates with hepatic interferon gene expression changes independent of cirrhosis. J Viral Hepat. 2016;23(7):496-505.

16. Meissner EG, Kohli A, Higgins J, Lee YJ, Prokunina O, Wu D, et al. Rapid changes in peripheral lymphocyte concentrations during interferon-free treatment of chronic hepatitis C virus infection. Hepatol Commun. 2017;1(7): 586-94

17. Osinusi A, Meissner EG, Lee YJ, Bon D, Heytens L, Nelson A, et al. Sofosbuvir and ribavirin for hepatitis $C$ genotype 1 in patients with unfavorable treatment characteristics: a randomized clinical trial. JAMA. 2013;310(8):80411.

18. Swaminathan S, Hu Z, Rupert AW, Higgins JM, Dewar RL, Stevens R, et al. Plasma interleukin-27 (IL-27) levels are not modulated in patients with chronic HIV-1 infection. PLoS One. 2014;9(6):e98989.
19. Danaher P, Warren S, Dennis L, D'Amico L, White A, Disis ML, et al. Gene expression markers of tumor infiltrating leukocytes. J Immunother Cancer. 2017;5:18.

20. Harrington PR, Fleischer R, Connelly SM, Lewis LL, Murray J. Ribavirin reduces absolute lymphocyte counts in hepatitis $C$ virus-infected patients treated with interferon-free, Direct-Acting Antiviral Regimens. Clin Infect Dis. 2015;61(6):974-7.

21. Robinson MW, Swann R, Sigruener A, Barclay ST, Mills PR, McLauchlan J, et al. Elevated interferon-stimulated gene transcription in peripheral blood mononuclear cells occurs in patients infected with genotype 1 but not genotype 3 hepatitis C virus. J Viral Hepat. 2014;22(4):384-90.

22. Rosenberg BR, Freije CA, Imanaka N, Chen ST, Eitson JL, Caron R, et al. Genetic variation at IFNL4 influences Extrahepatic interferon-stimulated gene expression in chronic HCV patients. J Infect Dis. 2018;217(4):650-5.

23. Lalle E, Bordi L, Caglioti C, Garbuglia AR, Castilletti C, Taibi C, et al. IFN-Alpha receptor-1 upregulation in PBMC from HCV naive patients carrying CC genotype. possible role of IFN-lambda. PLoS One. 2014;9(4):e93434.

24. Menezes EG, Coelho-Dos-Reis JG, Cardoso LM, Lopes-Ribeiro A, JonathanGoncalves J, Porto Goncalves MT, et al. Strategies for serum chemokine/ cytokine assessment as biomarkers of therapeutic response in HCV patients as a prototype to monitor immunotherapy of infectious diseases. Antivir Res. 2017:141:19-28.

25. Egli A, Santer DM, O'Shea D, Tyrrell DL, Houghton M. The impact of the interferon-lambda family on the innate and adaptive immune response to viral infections. Emerg Microbes Infect. 2014;3(7):e51.

26. Thomas E, Gonzalez VD, Li Q, Modi AA, Chen W, Noureddin M, et al. HCV infection induces a unique hepatic innate immune response associated with robust production of type III interferons. Gastroenterology. 2012;142(4): 978-88.

27. Obajemu AA, Rao N, Dilley KA, Vargas JM, Sheikh F, Donnelly RP, et al. IFNlambda4 attenuates antiviral responses by enhancing negative regulation of IFN signaling. J Immunol. 2017;199(11):3808-20.

28. Soza A, Everhart JE, Ghany MG, Doo E, Heller T, Promrat K, et al. Neutropenia during combination therapy of interferon alfa and ribavirin for chronic hepatitis C. Hepatology. 2002;36(5):1273-9.

29. Giorgini A, Capsoni F, Podda M, Lleo A, Battezzati PM, Ongari AM, et al. Treatment with PEG-interferon and ribavirin for chronic hepatitis $C$ increases neutrophil and monocyte chemotaxis. Ann N Y Acad Sci. 2009;1173:847-57.

30. Golden-Mason L, McMahan RH, Kriss MS, Kilgore AL, Cheng L, Dran RJ, et al. Early and late changes in natural killer cells in response to ledipasvir/ sofosbuvir treatment. Hepatol Commun. 2018;2(4):364-75.

31. Kramer B, Finnemann C, Sastre B, Lutz P, Glassner A, Wolter F, et al. IL-28B genetic variants determine the extent of monocyte-induced activation of NK cells in hepatitis C. PLoS One. 2016;11(9):e0162068.

32. Rogalska-Taranta M, Markova AA, Taranta A, Lunemann S, Schlaphoff $V$, Flisiak R, et al. Altered effector functions of NK cells in chronic hepatitis C are associated with IFNL3 polymorphism. J Leukoc Biol. 2015;98(2):283-94.

33. Boisvert $M$, Shoukry NH. Type III Interferons in hepatitis $C$ virus infection. Front Immunol. 2016;7:628.

34. Paolini R, Bernardini G, Molfetta R, Santoni A. NK cells and interferons. Cytokine Growth Factor Rev. 2015;26(2):113-20.

35. Fuertes Marraco SA, Neubert NJ, Verdeil G, Speiser DE. Inhibitory receptors beyond T cell exhaustion. Front Immunol. 2015;6:310.

36. Owusu Sekyere S, Suneetha PV, Kraft AR, Zhang S, Dietz J, Sarrazin C, et al. A heterogeneous hierarchy of co-regulatory receptors regulates exhaustion of HCV-specific CD8 T cells in patients with chronic hepatitis C. J Hepatol. 2015;62(1):31-40.

37. Benci JL, Xu B, Qiu Y, Wu TJ, Dada H, Twyman-Saint Victor C, et al. Tumor interferon signaling regulates a multigenic resistance program to immune checkpoint blockade. Cell. 2016;167(6):1540-54 e12.

38. Boussiotis VA. Molecular and biochemical aspects of the PD-1 checkpoint pathway. N Engl J Med. 2016;375(18):1767-78.

39. Mondelli MU. The multifaceted functions of ribavirin: antiviral, immunomodulator, or both? Hepatology. 2014;60(4):1126-9.

\section{Publisher's Note}

Springer Nature remains neutral with regard to jurisdictional claims in published maps and institutional affiliations. 